\title{
Equatorial deep jets triggered by a large vertical scale variability within the western boundary layer
}

\author{
by Marc d'Orgeville ${ }^{1,2}$, Bach Lien Hua ${ }^{1}$ and Hideharu Sasaki ${ }^{3}$
}

\begin{abstract}
The response of the deep equatorial ocean to an oscillatory baroclinic western boundary current is investigated in a continuously stratified primitive equation model. The symmetry of the current about the equator is such that mixed Rossby-gravity (MRG) waves are excited in the western part of the equatorial ocean. Depending on the forcing frequency, short to long scale (when compared to equatorial Rossby radius) monochromatic MRG waves are selected. The subsequent MRG wave destabilization generally leads to a much higher vertical mode response than the forced MRG wave mode.

In a channel, short MRG waves are destabilized by shear instability (Hua et al., 2007). In a basin, the destabilization occurs in the vicinity of the western boundary and leads to the formation of finite amplitude, nonlinear jets in the entire equatorial basin. The space and time pattern of the jets correspond to low-frequency oscillating equatorial basin-modes, the period of which is set by the dominant vertical mode of the response. The vertical scale of the jets is a function mainly of the forcing period and is independent of the forcing vertical mode, as long as the excited MRG waves are in a sufficiently short regime to be unstable.

As a result, an oscillatory western boundary current leads to a permanent equatorial zonal circulation, unlike a steady western boundary current. But most importantly, MRG wave destabilization appears to be a plausible formation mechanism for the observed Equatorial Deep Jets. The spatial and dynamical characteristics of the zonal circulation achieved with a 60-day forcing period are indeed compatible with the observations in the Atlantic Ocean.
\end{abstract}

\section{Introduction}

The distribution of deep tracer fields in the Atlantic Ocean reveals a connection between the thermohaline circulation and the equatorial ocean. At the depth of the North Atlantic deep water, Wüst (1935) first noted the presence of an oxygen maximum within the equatorial region relative to values in the interior ocean north and south of the equator, suggesting an intrusion from the western boundary layer of recently ventilated water masses.

1. Laboratoire de Physique des Océans (CNRS-IFREMER-UBO), IFREMER BP 70, 29280, Plouzané, France.

2. Present address: Atmospheric Physics Group, Department of Physics, University of Toronto, Toronto, Ontario, Canada.email:marcdo@atmosp.physics.utoronto.ca

3. Earth Simulator Center, Yokohama Institute for Earth Sciences, Japan Agency for Marine-Earth Science and Technology, 3173-25 Showa-machi, Kanazawa-ku, Yokohama 236-0001, Japan. 
Weiss et al. (1985) showed that a tongue of upper deep North Atlantic water spreads along the equator by tracing the CFC maximum path. At other depths, for water masses that do not carry CFCs, the same conclusion can be drawn from extrema of concentration of other tracers such as oxygen (Suga and Talley, 1995). The confinement of such extrema within a few degrees of the equator suggests the existence of an equatorial boundary layer that is connected to the thermohaline circulation, enabling recently ventilated water masses to penetrate into the basin interior along the equator.

The meridional and zonal structure of an equatorial boundary layer in a linear model depends on the diffusion/dissipation that is used. For a linear diffusion (Kawase, 1987) the meridional scale is $L_{y}=R_{d}(\kappa / \lambda)^{1 / 4}$, where $\kappa$ is diffusion and $\lambda$ is dissipation, and the zonal structure is exponentially confined to the western boundary within a zonal scale $L_{x}=R_{d} \sqrt{\beta^{2} / \kappa \lambda}$ (Wang, 1995), where $R_{d}$ is the equatorial radius of deformation. For a strong Laplacian diffusion (Furue, 2001), the characteristic meridional scale corresponds to a Stommel or Munk frictional boundary layer scale and the zonal structure linearly decreases from the western to the eastern boundary. In both cases, an equatorial trapping and thus the existence of an equatorial boundary layer is fundamentally linked to a strong diffusion. Paradoxically, increasing the diffusion causes a decrease in amplitude of the equatorial zonal velocity in the interior (Wang, 1995). None of the presently published GCM solutions significantly deviates from Kawase's linear model and advective effects remain weak in the deep equatorial ocean interior.

To date, no numerical model has succeeded in faithfully reproducing the observed deep zonal equatorial circulation. The latter is dominated in the three equatorial oceans by a vertical stacking of zonal jets of alternating directions along the equator (Luyten and Swallow, 1976; Leetma and Spain, 1981; Eriksen, 1982). Their characteristic vertical scale is well established for a given ocean (around $500 \mathrm{~m}$ in the Atlantic, $350 \mathrm{~m}$ in the Pacific and $600 \mathrm{~m}$ in the Indian Ocean, see respectively, Gouriou et al., 1999; Firing, 1987; Dengler and Quadfasel, 2002). In the Atlantic, the important transport by the deep jets (10-20 Sv eastward, Schmid et al., 2005) could play a non-negligible role in the equatorial intrusion from the western boundary layer. At the depth of the Upper North Atlantic Deep Water, eastward jets correspond to a maximum in CFC within the equatorial ocean in the western (Andrie et al., 1998), central (Gouriou et al., 2001) and eastern (Bourles et al., 2003) parts of the basin, suggesting a direct connection with the western boundary layer. Existing observations do not allow conclusion as to their temporal behavior; some studies tend to show either a stationary or an intermittent behavior (Send et al., 2002; Bunge et al., 2006) while other studies indicate a very low-frequency vertical propagation (Johnson et al., 2002; Johnson and Zhang, 2003). The low-frequency variability suggests an interpretation in terms of long equatorial Rossby or Kelvin waves. However observations also unambiguously demonstrate the fundamentally nonlinear character of the deep jets with local Froude and Rossby number values larger than 0.5 for all available data (as discussed in Section 5). The so-called Equatorial Deep Jets (EDJs) formation mechanism and their low-frequency variability remain to be elucidated completely. Formation mechanisms must be able to first give, a rationale 
of the vertical scale selection and second, the source of the nonlinear dynamics that impact the potential vorticity distribution (Hua et al., 1997).

The deep equatorial ocean is also characterized by a strong intraseasonal variability ( 15 to 60 days). The latter is mainly seen in fluctuations of the meridional velocity at the equator, can be coherent on vertical scales larger than 1000 meters, and can exceed $20 \mathrm{~cm} \cdot \mathrm{s}^{-1}$ (Weisberg and Horigan, 1981; Bunge et al., 2006). The spatial and temporal structure of such oscillations generally appears compatible with the dynamics of a free mixed Rossbygravity (MRG) wave. Given that far from the surface and western boundary layers, one possible source of energy that is clearly identified comes from propagating free equatorial waves, this variability could play a non-negligible role in the deep equatorial dynamics: this is a key underlying assumption of the present work (see also d'Orgeville et al., 2004). In the case of an equatorial channel, Hua et al. (2007) have shown that monochromatic MRG waves can destabilize while generating a steady zonal circulation of significantly smaller vertical scale. The first question addressed in this paper is: can large vertical scale MRG waves be responsible for the formation of small vertical scale equatorial zonal jets? Compared to channel results this question can be recast as: how MRG waves destabilization modified in a basin geometry, i.e. in the presence of zonal boundaries?

Moreover, observations show a significant intraseasonal variability of western boundary currents. In the Atlantic Ocean, at the equator, oscillation periods range from 30 to 60 days at the depths of North Atlantic Deep Waters (Johns et al., 1993; Fischer and Schott, 1997), Intermediate Waters (Richardson and Fratantoni, 1999) and surface layers (Johns et al., 1990). Deep western boundary current variability is also found at intraseasonal time scales in the other oceans. For example, a striking 59-day peak exists at $20 \mathrm{~S}$ in the Indian Ocean (Warren et al., 2002), and a period of 4 months at $18 \mathrm{~N}$ is documented in the Pacific Ocean (Kawabe et al., 2005). This western deep variability can be a source of MRG waves in the equatorial ocean, and therefore, the second question that is addressed in this paper is: can a time-oscillating western boundary current induce a persistent equatorial circulation? This situation has to be contrasted with the steady case where only a transient equatorial circulation is achieved (Kawase et al., 1992).

These questions prompted us to force a primitive equation equatorial basin model by an oscillating inter-hemispheric meridional velocity within the western boundary layer, which excites MRG waves along the equator. The model is continuously stratified and fully nonlinear to enable interactions among different vertical modes which come into play in the MRG waves destabilization. These interactions are fundamental to the mechanism presented here. In previous studies about temporal variability impact on equatorial circulation, these interactions were not present because of the use of a 1-layer shallow water model (Thompson and Kawase, 1993; Li et al., 1996), the assumption of linearity (Ponte, 1989), or the lack of a sufficiently high vertical model resolution (Li and Chang, 1999).

This paper provides evidence that an oscillating western boundary current can induce a persistent equatorial circulation, in the form of highly nonlinear equatorial jets with small vertical scales. The last question addressed in this study is: how is the vertical scale of 
those jets selected? Results of the analytic model predicting jet vertical scales in a channel (Hua et al., 2007) will be confirmed here by numerical simulations in a basin.

This paper is organized as follows. After recapitulating the results on the destabilization of MRG waves in a channel, Section 2 motivates the MRG wave regime which has been chosen for application to the observations in the equatorial Atlantic. In Section 3, we present the idealized configuration of the model where the excitation of monochromatic MRG waves is produced by an extra-equatorially forced oscillating western boundary current. Section 4 answers questions about MRG wave evolution in a basin geometry and the impact of an oscillating western boundary current on the equatorial ocean by describing MRG wave destabilization in a basin and the temporal behavior of the resulting jets. Section 5 then compares the dynamical and spatial characteristics of the simulated jets with those of the observed EDJs in the Atlantic Ocean, after addressing the question of their vertical scale selection. Finally, Section 6 summarizes all results and discusses their application to observations.

\section{Destabilization of mixed Rossby-gravity waves}

\section{a. Equatorial channel geometry (Hua et al., 2007)}

MRG waves are antisymmetric ${ }^{4}$ waves that have a maximum in potential vorticity anomaly ${ }^{5}$ at the equator when compared to other antisymmetric free waves of comparable Froude number $\frac{V^{\star}}{c^{\star}}$, where $V^{\star}$ is the maximum wave amplitude in meridional velocity and $c^{\star}$ is the long gravity wave speed for the vertical mode of the MRG wave. Hua et al. (2007) have shown that a monochromatic westward propagating MRG wave is unstable.

In a zonally periodic channel with an initial value problem, two types of destabilization have been found depending on the MRG wave zonal wavenumber regime. Long westward MRG waves $\left(\hat{k} \equiv \frac{k^{\star}}{R_{d}} \gtrsim-2\right.$, where the zonal wavenumber $k^{\star}$ is nondimensionalized by the equatorial radius of deformation $R_{d} \equiv \sqrt{\frac{c^{\star}}{\beta}}$ ) undergo a slow destabilization through weakly nonlinear resonant triad interactions and the initial MRG wave survives the nonlinear interactions in the end state. In contrast, short westward MRG waves $\left(\hat{k} \equiv \frac{k^{\star}}{R_{d}} \lesssim-2\right)$ are subject to a strong and rapid destabilization where the initial MRG wave is completely suppressed in the end state dominated by zonally symmetric barotropic and strongly baroclinic jets.

This is illustrated by instantaneous zonal velocity after 150 days of an initialized MRG wave in a channel geometry (Fig. 1). While for case $\hat{k}=-1.5$ the MRG wave is almost unperturbed, for case $\hat{k}=-3$ the MRG wave has already undergone a strong destabilization which leads to strongly baroclinic jets. This second type of destabilization due to shear

4. Througout the paper, equatorial symmetry/antisymmetry refers to the usual convention for equatorial free waves, i.e. a wave is symmetric (resp. antisymmetric) if the zonal velocity and the density fields are symmetric (antisymmetric) and the meridonnal velocity field is antisymmetric (symmetric).

5. $P V \propto v / \omega$, where $v$ is the meridional velocity component and $\omega$ is the wave frequency (Ripa, 1981). 

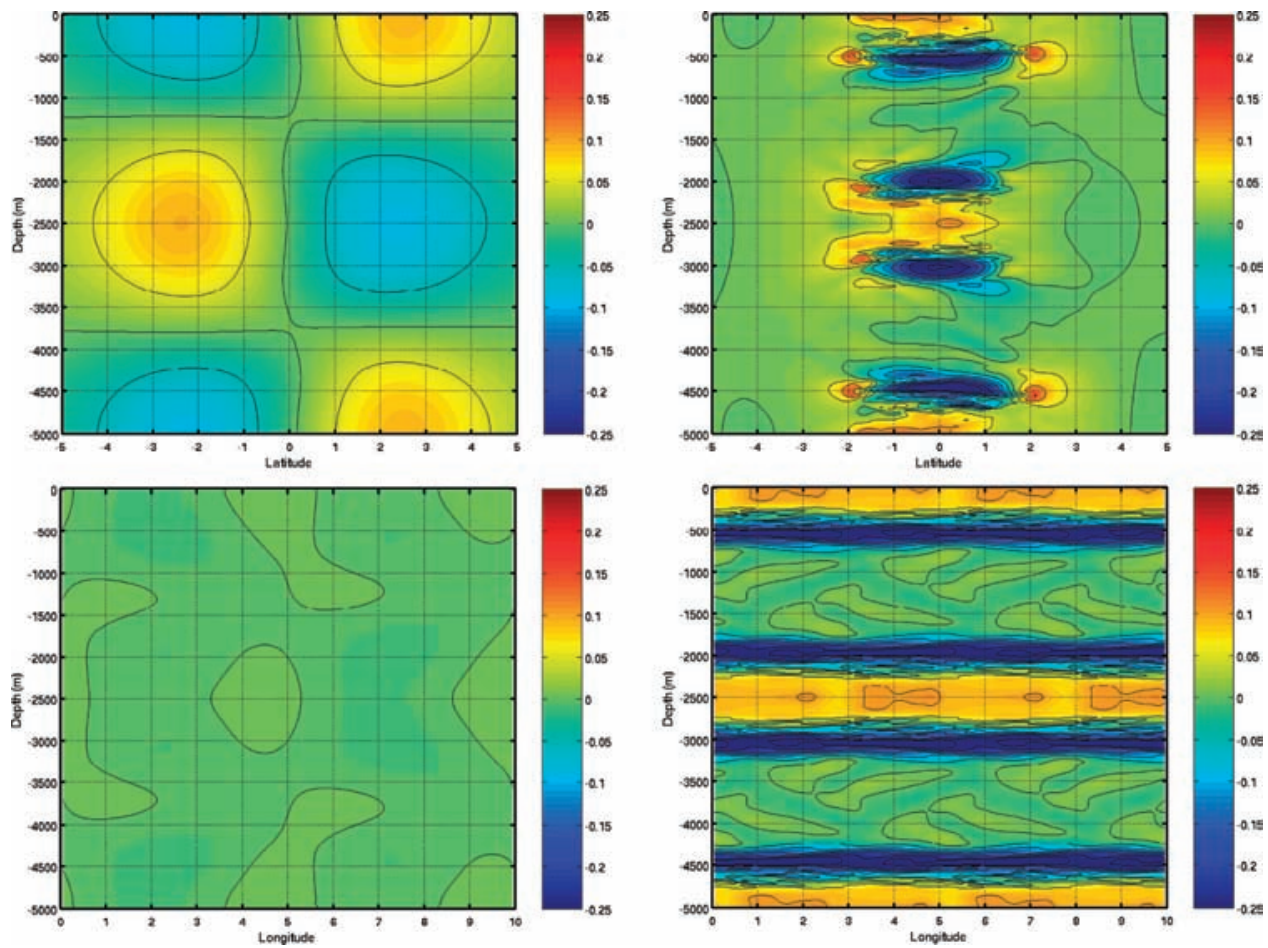

Figure 1. Initial value simulations of a MRG wave in a periodic channel from Hua et al. (2007). (Top) Depth-latitude instanteneous distribution of zonal velocity. (Bottom) Depth-longitude instantaneous distribution of zonal velocity along the equator. Both cases $(\hat{k}=-1.5$ on the left, $\hat{k}=-3$ on the right) correspond to an initial vertical mode of the MRG wave $n^{\star}=2$ and an initial Froude number $\mathrm{Fr}=0.2$.

instability is the most interesting case in terms of strongly baroclinic zonal jet formation. The following Sections will study this as an EDJs formation mechanism.

\section{b. Equatorial basin geometry}

In a basin, an important parameter for the destabilization of MRG waves will be the ratio of the characteristic time of propagation across the basin $\tau_{P}$ to the characteristic destabilization time scale $\tau_{D}$,

$$
\Gamma=\frac{\tau_{P}}{\tau_{D}} .
$$

The larger $\Gamma$ the longer time the wave will have to destabilize before reaching the other meridional boundary. This is analogous to the destabilization of mid-latitude long Rossby waves emanating from the eastern meridional boundary of the basin (LaCasce and Pedlosky, 2004). 
For a monochromatic MRG wave of wavenumber $k^{\star}$, the group velocity $c_{g}\left(k^{\star}\right)$ is eastward and decreases with $-k^{\star}$. The characteristic time $\tau_{P}$ will be an increasing function of $-k^{\star}$ and of the basin zonal extent $L_{B}$,

$$
\tau_{P}=\frac{L_{B}}{c_{g}\left(k^{\star}\right)} .
$$

In a channel, the growth rate of the MRG wave instability is found to be a growing function of the initial MRG wave Froude number $F r$ and its zonal wavenumber $-k^{\star}$. The characteristic time for destabilization is consequently a decaying function of $\mathrm{Fr}$ and $-k^{\star}$,

$$
\tau_{D}\left(F r, k^{\star}\right) .
$$

$\Gamma$ is an increasing function of $F r,-k^{\star}$ and $L_{B}$. As a result, in a basin, MRG wave destabilization will be favored by long zonal extent, short waves and large amplitude.

This paper aims to provide an application to the observed EDJs in the Atlantic Ocean. Even if the Atlantic Ocean has a relatively short basin length, Sections 4 and 5 will show that MRG waves of realistic scales (time, length and velocity scales) always have the time to be destabilized, if they are sufficiently short. $\tau_{P}$ and $\tau_{D}$ will be explicitly given for the specific case described in Section 4. A discussion of the Pacific and Indian ocean EDJs will be provided at the end of the paper.

\section{Setup and forcing of the primitive equations model}

Although deep equatorial observations exhibit a large spectrum of intraseasonal frequencies (e.g. Bunge et al., 2006), we restrict ourselves to the impact of monochromatic MRG waves on the zonal circulation of an equatorial basin. The designed idealized problem (rectangular basin, homogeneous stratification, monochromatic waves) allows a direct comparison with Hua et al. (2007)'s results in a channel.

PE models used in this study are described in the first subsection. The forcing that excites MRG waves in the basin, while allowing the equatorial ocean response free to adjust, is explained in the next subsection.

\section{a. Numerical model}

In Section 4, numerical simulations are presented to answer the questions about MRG wave evolution in a basin geometry and the impact of an oscillating western boundary current on the equatorial ocean. These questions require relatively low vertical and horizontal resolution simulations. This was performed with the vectorized code SPEM (Haidvogel et al., 1991), on a single processor of a NEC SX5 supercomputer. In Section 5, where the characteristics of the obtained zonal equatorial jets are compared with observations, the issue of their vertical scale necessitated very high resolution, both horizontally and vertically. These simulations required the use of a vectorized and highly-parallelized code, 
ROMS (Shchepetkin and McWilliams, 2005), on 64 processors at the Earth Simulator. Thus, two different models were used in this study but they both resolve the primitive equations in Cartesian coordinates with the Boussinesq and hydrostatic approximations. They differ only by the rigid lid approximation in SPEM versus the free surface in ROMS. Results discussed here are qualitatively independent of models and/or resolutions used.

The geometry used in the numerical experiments is a flat bottom, rectangular basin that extends from $15 \mathrm{~N}$ to $15 \mathrm{~S}$ in latitude and from 0 to $5000 \mathrm{~m}$ depth. The zonal extent of the basin is $30^{\circ}$ in longitude. The grid resolution of $1 / 4^{\circ}$ in SPEM and $1 / 11^{\circ}$ in ROMS is isotropic in the horizontal. Vertical resolution from surface to bottom is $50 \mathrm{~m}$ for SPEM and 25 or $12.5 \mathrm{~m}$ for ROMS.

A Laplacian horizontal dissipation is applied near the western, northern and southern boundary. It has a maximum amplitude of $1000 \mathrm{~m}^{2} \mathrm{~s}^{-1}$ in the western boundary layer. It is also increased to $10000 \mathrm{~m}^{2} \mathrm{~s}^{-1}$ poleward of $12 \mathrm{~N}$ and $12 \mathrm{~S}$, to create sponge layers. This Laplacian horizontal dissipation decreases exponentially to zero in the interior with a zonal scale of $2.5^{\circ}$ from the western boundary. A vertical Laplacian dissipation/diffusion is applied on the whole basin with a constant amplitude of $10^{-4} \mathrm{~m}^{2} \mathrm{~s}^{-1}$ in SPEM and $10^{-6} \mathrm{~m}^{2} \mathrm{~s}^{-1}$ in ROMS. For numerical stability, a weak horizontal fourth-order hyper-diffusion/dissipation of amplitude $4.10^{10} \mathrm{~m}^{4} \mathrm{~s}^{-1}$ is applied to the three prognostic equations in SPEM, and a weak horizontal laplacian diffusion/dissipation of amplitude $70 \mathrm{~m}^{2} \mathrm{~s}^{-1}$ is used in ROMS.

The density field is initialized to a depth dependent density $\rho=\bar{\rho}(z)$ with a constant Brunt-Vaisala frequency $N=\sqrt{-\frac{g}{\rho_{0}} \frac{d \bar{\rho}}{d z}}=2 \cdot 10^{-3} \mathrm{~s}^{-1}$, a value characteristic of deep oceanic conditions. Poleward of $12 \mathrm{~N}$ and $12 \mathrm{~S}$, the density is restored to the initial density $\bar{\rho}$ plus the baroclinic forcing perturbation density $\tilde{\rho}$ defined below. The restoring time scale is $1 / r_{\rho}=10$ days.

Vertical boundary conditions at the top and bottom are $\frac{\partial u}{\partial z}=\frac{\partial v}{\partial z}=\frac{\partial \rho}{\partial z}=0$. Horizontal boundary conditions are $u=v=0$ and $\frac{\partial \rho}{\partial x}=0$ at the eastern and western boundaries, $u=0, v=\tilde{v}$ and $\rho=\bar{\rho}+\tilde{\rho}$ at the northern and southern boundaries, where $\tilde{v}$ and $\tilde{\rho}$ are the forcing fields defined below. ${ }^{6}$

\section{b. Extra-equatorial forcing within the western boundary layer}

In order to excite MRG waves in the basin, while leaving the equatorial ocean response free to adjust, a baroclinic, temporally oscillating mass flux that is confined to the western boundary is applied at its northern and southern boundaries through

$$
\tilde{v}\left(15^{\circ}\right)=\tilde{v}\left(-15^{\circ}\right)=V(x, z) \cos \left(\omega^{\star} t\right)
$$

with

$$
V(x, z)=A^{\star} G^{\prime}(x) H(z) .
$$


In sponge layer regions (poleward of $12 \mathrm{~N}$ and $12 \mathrm{~S}$ ), the density field is restored toward a prescribed perturbation $\tilde{\rho}$ in geostrophic balance with the boundary conditions $\tilde{v}$, i.e.

$$
\tilde{\rho}=-A^{\star} \frac{\rho_{0}}{g} \beta y G(x) H^{\prime}(z) .
$$

The zonal structure ${ }^{7}$ is defined as

$$
G(x)=-\lambda_{x} \exp \left(-\frac{x^{2}}{2 \lambda_{x}^{2}}\right), \quad G^{\prime}(x)=\frac{x}{\lambda_{x}} \exp \left(-\frac{x^{2}}{2 \lambda_{x}^{2}}\right),
$$

with $\lambda_{x}=1^{o}$. The vertical structure is defined as

$$
H(z)=\cos \left(m^{\star} z\right), \quad H^{\prime}(z)=-m^{\star} \sin \left(m^{\star} z\right),
$$

where $m^{\star}$ is the prescribed forcing vertical mode.

Because of the sinusoidal vertical structure of the forcing, the global mass of the basin is constant in time. Since this forcing is antisymmetric, at a given depth the mass which enters in one hemisphere exits from the other. The volume of a density layer is therefore steady in time, and an interhemispheric mass flux takes place as an oscillating western boundary current at the equator. The consequences of a symmetric forcing (i.e. $\tilde{v}\left(15^{\circ}\right)=-\tilde{v}\left(-15^{\circ}\right)$ ) is shortly described in Section $4 \mathrm{~d}$. In that case, the global mass is still steady in time, but the volume of a given density layer oscillates in time.

For antisymmetric forcing, another way to understand it can be that each oscillating mass source, to the north and the south, forces a coastal Kelvin wave which propagates along the western boundary towards the equator. Because the forcing is antisymmetric, the northern and southern coastal Kelvin wave signals will add up when the waves reach the equator and thus create an interhemispheric mass flux that oscillates with frequency $\omega^{\star}$ at the western boundary. Such oscillation of the meridional velocity component within the western boundary at the equator excites MRG wave trains which propagate eastward along the equator with the wave train group velocity.

For all simulations, the vertical mode of the forcing is chosen as $m^{\star}=n^{\star} \pi / h_{0}$ where the total depth $h_{0}=5000 \mathrm{~m}$. The linearly excited equatorial response is also of vertical mode $m^{\star}$, with a long gravity wave speed $c^{\star} \simeq 3.2 / n^{\star} \mathrm{m} \mathrm{s}^{-1}$ and the equatorial deformation radius is $R_{d}=\sqrt{c^{\star} / \beta}$. The characteristics of the monochromatic waves that are excited at the equator are thus functions of the frequency and amplitude. The frequency $\omega^{\star}$ determines the zonal wavenumber of the MRG waves through the dispersion relation

$$
k^{\star}=\frac{\omega^{\star}}{c^{\star}}-\frac{\beta}{\omega^{\star}} .
$$

The forcing amplitude $A^{\star}$ permits variation of the Froude number $F r=\frac{V^{\star}}{c^{\star}}$, where $V^{\star}$ is a posteriori measured in the numerical simulation at the equator. 
Table 1. SPEM-simulations forcings have a vertical mode $n^{\star}=2$ and a 40 -day period. This vertical mode corresponds to a long gravity wave speed of $c^{\star} \simeq 1.6 \mathrm{~m} \mathrm{~s}^{-1}$ and an equatorial deformation radius $R_{d}=\sqrt{c^{\star} / \beta} \simeq 260 \mathrm{~km}$. In SW and SW2, the excited MRG wave nondimensional zonal wavenumber is $\hat{k}=-3$. SYM has the same extra-equatorial forcing amplitude $A^{\star}$ as SW2.

\begin{tabular}{lcc} 
Simulation & $F r=V^{\star} / c^{\star}$ & Forcing \\
\hline SW & 0.2 & antisymmetric \\
SW2 & 0.4 & antisymmetric \\
SYM & - & symmetric
\end{tabular}

\section{Equatorial response in a basin geometry}

In this section we explore the effect of a basin geometry on the destabilization of MRG waves to answer the questions posed in the introduction: can large vertical scale MRG waves be responsible for the formation of small vertical scale equatorial zonal jets? Can a time-oscillating western boundary current induce a persistent equatorial circulation?

The parameters for all the simulations presented in this Section are summarized in Table 1. The Froude number is computed from the characteristics of the waves that are excited in simulations where the equations of motion have been linearized. Instantaneous equatorial zonal circulations obtained are plotted in Figure 2.
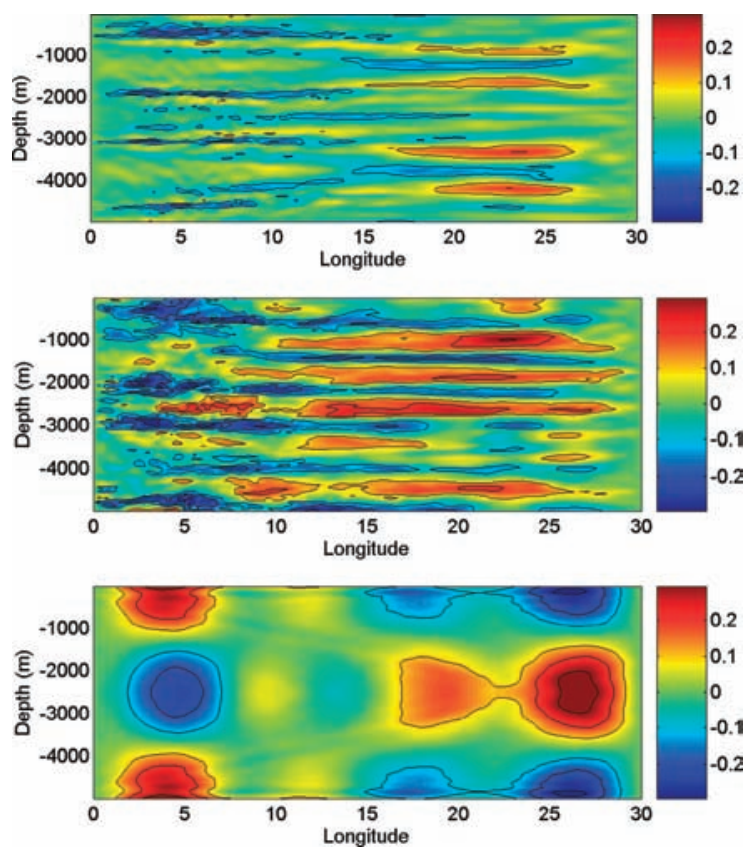

Figure 2. Instantaneous distribution of zonal velocity along the equator for SW (top), SW2 (middle) and SYM (bottom), after 6 years of simulations. Solid contours every $10 \mathrm{~cm} \cdot \mathrm{s}^{-1}$ except 0 . 

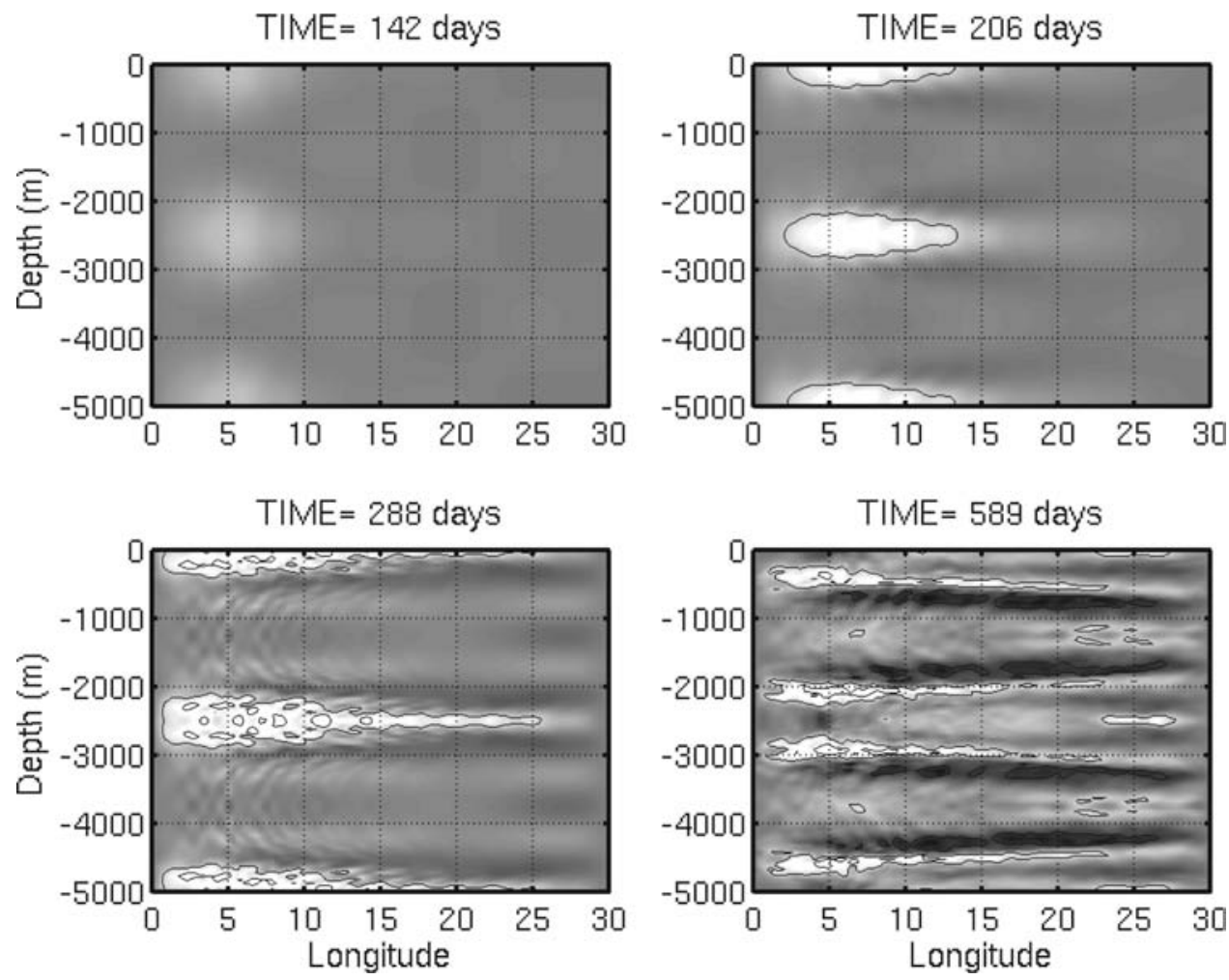

Figure 3. Instantaneous distribution of zonal velocity along the equator for simulation SW.

In a first subsection the MRG wave destabilization in a basin geometry is described during the spinup of the moderately nonlinear simulation SW. The temporal behavior of the jets in terms of equatorial basin modes is then demonstrated for simulation SW. Other effects specific to the basin geometry - for instance, the influence of the MRG wave nonlinear regime - are discussed next. In the last subsection, MRG wave destabilization is contrasted to the case of stable Kelvin waves excited by a symmetric forcing.

\section{a. Spinup and localization of MRG wave destabilization}

The spinup of simulation SW shows that a very strong destabilization of MRG waves occurs, and the waves never reach the eastern boundary. This destabilization is predictable (Section 2b): from linear theory, the basin-crossing time of the MRG waves is $\tau_{P}=480$ days, whereas the growth rate of the instability, deduced in a channel, gives $\tau_{D} \sim 6$ days $^{8}$. Zonal and meridional velocity distributions on the equator (Figs. 3,4 ) reveal

8. $\tau_{D}=\mu^{-1}$ with the growth rate $\mu$ computed as $\mu=\hat{\mu}\left(V^{\star} / R_{d}\right)$, where $\hat{\mu} \simeq 1.5$ is the nondimensional growth rate found by Hua et al. (2007). 

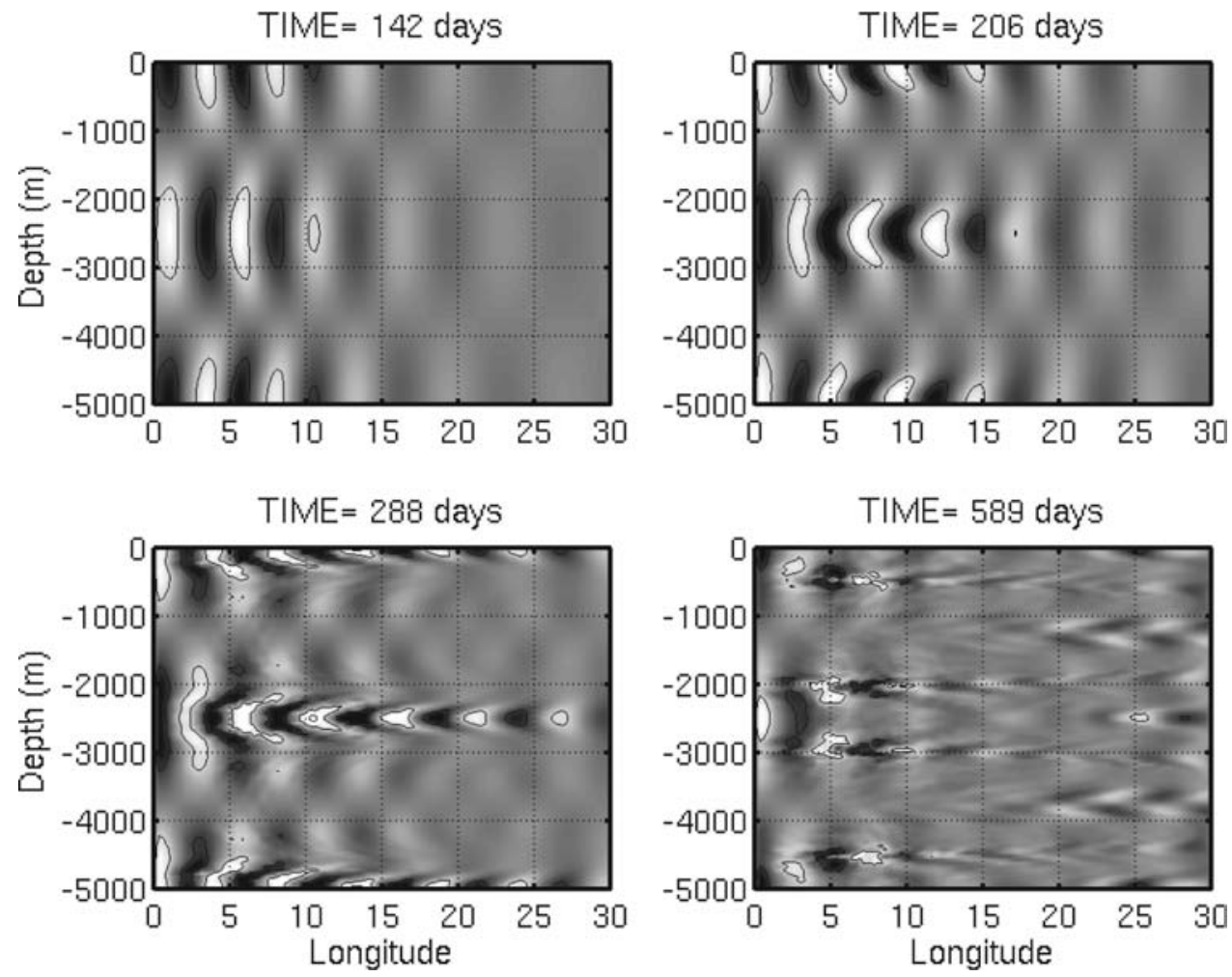

Figure 4. Instantaneous distribution of meridional velocity along the equator for simulation SW.

that the destabilization process is strongly localized in the vertical at the depth of extrema of meridional velocity of the MRG wave trains (at the surface, at mid-depth and at the bottom) near the western boundary.

At $t=142$ days the MRG wave trains excited by the forced baroclinic western boundary current are visible in meridonal velocities (Fig. 4a) since they have no zonal velocities at the equator (Fig. 3a). Strong destabilization is not yet seen at this time. The MRG waves seem to follow linear propagation theory with a vertical mode $n^{\star}=2$ imposed by the forcing (Fig. 4a).

At mid-depth $(2500 \mathrm{~m})$, the arrival of the MRG wave train at the equator triggers the build up of a westward current in the western part of the basin (Fig. $3 \mathrm{~b}, t=206$ days). Its vertical scale decreases with time, while its amplitude increases up to $30 \mathrm{~cm} \cdot \mathrm{s}^{-1}(t=288$ days). Two eastward currents, located right above and beneath this westward jet, are then formed and the whole pattern extends to the east $(t=288$ days). The reflection of this signal at the eastern boundary triggers Rossby waves with different vertical scales, which extinguish the initial westward jet and lead to the creation of two eastward currents at $z=1250$ and $3750 \mathrm{~m}$ (between $20^{\circ}$ and the eastern boundary, Fig. 3c), which propagate westward. In 
the western part of the basin the initial westward jet at $2500 \mathrm{~m}$ disappears and is replaced by eastward and westward zonal jets of smaller vertical scale (Fig. $3 \mathrm{~d}, t=589$ days). Such small vertical scales gradually fill the whole basin through a complex combination of large-amplitude long Kelvin and Rossby waves.

After this initial phase, MRG waves that are excited remain confined to the western boundary on a zonal scale that is comparable to the MRG wavelength (e.g. vertical mode 2, visible in the western part of the basin, Fig. 4d). The extra-equatorial forcing permanently excites these MRG waves in the equatorial region, but their destabilization is confined near the western boundary and concurrently forces unsteady zonal jets of small vertical scales in the whole basin (Fig. 3d).

Finally, the vertical mode of the jets obtained through the destabilization of MRG waves in the basin is visually the same as the one obtained in the initial-value problem in the channel (mode 8 in Figs.1-right and 2-top). But other smaller vertical scales are also clearly present in simulation SW. Vertical mode spectrum reveals that modes 8 and 12 can reach the same maximum amplitude (Fig. 7). As demonstrated by Hua et al. (2007), this instability develops with the growth and saturation of zonally symmetric Rossby and Kelvin waves with distinct vertical modes (mainly 8 and 12 for the case $\hat{k}=-3$ ). In a periodic channel, initial value simulations show that (i) in the case of a periodic initial MRG wave the final circulation is dominated by vertical mode number 8 , whereas (ii) in a non-periodic case both vertical modes 8 and 12 grow. In the basin geometry, due to reflection on the eastern and western boundaries, both modes 8 and 12 are present in the whole basin.

More importantly, unlike the channel case where these jets can be stationary because of the absence of meridional boundaries, the jets that are obtained in the basin geometry are unsteady. This temporal behavior is discussed below.

\section{b. Equatorial jets and basin modes}

The zonal structure of the nonlinear jets in simulation SW is unsteady. The projection on standing vertical modes of the zonal velocity at the equator allow us to better visualize and understand its temporal evolution. For instance, vertical mode 8 shows regular temporal oscillations with a period of about 420 days (Fig. 5). This period corresponds roughly to the linear theoretical prediction of the first equatorial basin mode of this vertical mode

$$
T_{8}=\frac{4 L_{B}}{c_{8}} \approx 385 \text { days }
$$

where $c_{8}=N h_{0} / 8 \pi$ is the long gravity wave speed of vertical mode 8 . These equatorial basin modes have been analytically derived in the limit of long waves on a semi-infinite equatorial beta plane (Cane and Moore, 1981), and have been numerically confirmed for an equatorial basin of finite meridional extent (Yang and Liu, 2003). For a given baroclinic mode of separation constant $c_{n}$, such basin modes can roughly be explained as equatorial Kelvin and long Rossby wave propagation. A Kelvin wave propagates eastward across the 


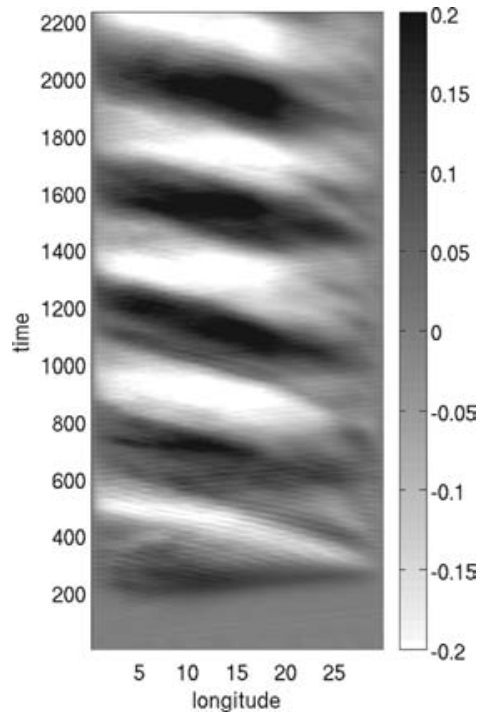

Figure 5. Time-longitude diagram of the projection of equatorial zonal velocities on vertical mode 8 in simulation $\mathrm{SW}$.

basin in a time $\frac{L_{B}}{c_{n}}$. At the eastern boundary, this Kelvin wave is reflected as a long Rossby wave of meridional mode 1 . This Rossby wave propagates westward across the basin in a time $3 \frac{L_{B}}{c_{n}}$. At the western boundary, this Rossby wave is eventually reflected as a Kelvin wave, and so on.

This propagation of vertical mode 8 can be seen on longitude/time diagram (Fig. 5). Westward propagation of Rossby waves are visible in the whole basin and the Kelvin wave eastward propagation is visible mostly at the start of the simulation and at the time of the reflections occurring near the western boundary. The difference between the theoretical period and the one estimated from the numerical simulation could be due to the nonlinear character of the structures that are propagating as well as to the variability of other vertical modes present in the simulation. Another possible explanation is the finite meridional length of the basin. Indeed equatorial basin modes that have been numerically determined in this case, have a slightly longer period than those predicted analytically (Yang and Liu, 2003). This is mostly due to the presence of northern and southern boundaries where sponge layers dissipate the coastal Kelvin wave signal.

Finally, it must be emphasized that the vertical scale selection (discussed in Section 5a) is independent of the basin zonal length scale, as seen in simulations with longer basin zonal length (not shown). The presence of meridional boundaries and the permanent forcing in the basin simulation appear only to prevent the existence of a steady circulation and to favor forced low-frequency equatorial basin modes of the selected vertical modes instead. 


\section{c. Other effects in a basin geometry}

The temporal evolution of the total equatorial zonal velocity as a function of depth reveals the dominant presence of the standing vertical mode 8 that oscillates with the same period as the previously described basin mode (Fig. 6). If the excited MRG wave amplitude is increased (Fig. 6a, simulation SW, to Fig. 6b, simulation SW2), temporal variability of the jets remains low-frequency but becomes dominated by vertical propagation. For Rossby or Kelvin type waves, energy propagates upward when the phase propagates downward (and vice versa). Since the meridional structure of the jets are either of Kelvin or Rossby type, an energy divergence is indicated at the depths of maximum forcing (surface, $2500 \mathrm{~m}$, bottom), while convergence occurs at depths of forcing nodes (1250 and $3750 \mathrm{~m}$ ). This corresponds to an energy radiation from levels where the instability of MRG waves is vertically localized to levels where no instability occurs.

The flow is thus no longer dominated by standing vertical modes in SW2 compared to SW. Numerous other vertical scales are present in the whole basin and the vertical scale of the jets does not seem to be the same (Fig. 2). This is confirmed by vertical mode spectra of the zonal velocity at the equator (Fig. 7): for SW, the vertical scales of standing modes 8 and 12 dominate the zonal equatorial circulation while the spectrum of simulation SW2 presents a much richer distribution centered around vertical mode 12. As explained previously, both mode 8 and 12 result from this instability. However, depending on the nonlinear regime of the MRG waves, the relative amplitude between them can change. Therefore, for simulations
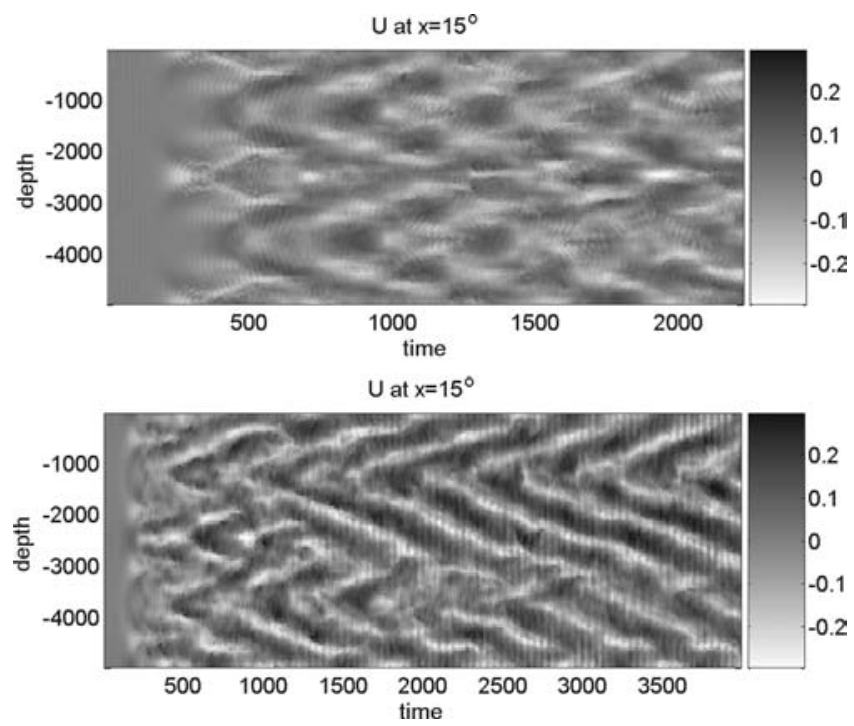

Figure 6. Time-depth diagram of equatorial zonal velocity at the equator for $x=15^{\circ}$ in simulation SW (top) and SW2 (bottom). 

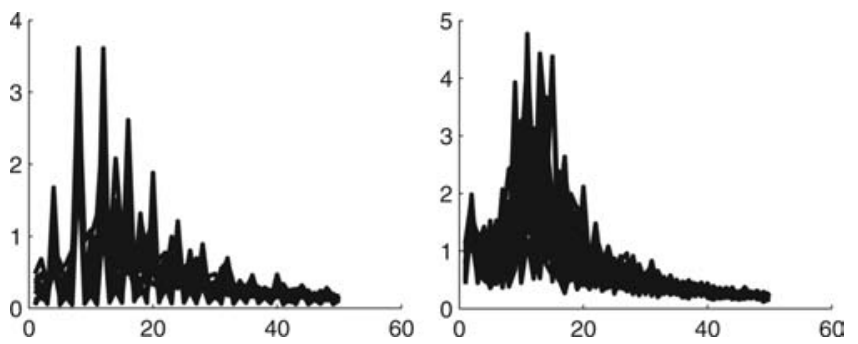

Figure 7. Zonally averaged vertical mode spectra of zonal velocity at the equator for simulations SW (left) and SW2 (right) at different times.

SW and SW2, the jets vertical modes are 4 to 6 times the forcing vertical mode, as predicted in channel geometries.

Finally, for simulation SW2, every dominant vertical mode between 8 to 16 has a time scale that agrees roughly with its period of first equatorial basin mode $T_{n}=4 L_{B} / c_{n}$. The time variability of the jets seems therefore to depend on their selected vertical scales (then on the Froude number $F r$ of the excited MRG waves) and on the basin length $L_{B}$. The prediction of this time scale is difficult since it also depends on the basin mode number: for simulation SW, the vertical mode 12 presents a horizontal pattern (not shown) corresponding to a second equatorial basin mode, i.e. with one node in the middle of the basin and a period $2 L_{B} / c_{n}$.

\section{d. Stability of Kelvin waves}

At any given frequency and vertical mode, numerous kinds of equatorial waves can be excited. If instead of the previous antisymmetric forcing, a symmetric one is applied (simulation SYM), only Kelvin waves are excited in the equatorial waveguide. The achieved equatorial circulation in SYM is strikingly different from SW2 (Fig. 2b-c), whereas southern and northern sources have the same amplitude in both simulations. The difference between the two cases is due to the fact that Kelvin waves are stable while MRG waves destabilize through shear instability. Therefore in SYM, the equatorial response has the same vertical mode and the same period as the forcing. In contrast, in SW2 there is a transfer from the forced low vertical mode intraseasonal variability to low frequency high vertical mode zonal jets. The interhemispheric character of the oscillating western boundary current and the subsequently excited equatorial oscillating zonal shear, $\frac{\partial V}{\partial x}$, are thus fundamental to obtained high vertical mode equatorial jets.

\section{Atlantic application}

In the simulations presented in the previous Section, the instantaneous circulation is dominated by a vertical stacking of zonal jets of alternating directions along the equator, thus reproducing the main characteristics of the available observations in the equatorial ocean. As highlighted in the introduction, a critical issue with respect to the EDJs is the ability 
Table 2. ROMS-simulations forcings have a vertical mode $m^{\star}=\pi n^{\star} / h_{0}$ and a $T^{\star}$ days period. The nondimensional zonal wavenumber of the excited MRG waves is $\hat{k}$. The zonal jets vertical mode obtained in the simulation is $p_{\text {jets }} \equiv p_{J}^{\star} \pi / h_{0}$ (see text and Fig. 9).

\begin{tabular}{cccc|cccc|cccc}
$\boldsymbol{T}^{\star}=\mathbf{4 0}$ & $p_{\text {jets }}$ & $n^{\star}$ & $\hat{k}$ & $\boldsymbol{T}^{\star}=\mathbf{5 7}$ & $p_{\text {jets }}$ & $n^{\star}$ & $\hat{k}$ & $\boldsymbol{T}^{\star}=\mathbf{7 4}$ & $p_{\text {jets }}$ & $n^{\star}$ & $\hat{k}$ \\
\hline M1T40 & $\mathbf{8 - 1 2}$ & 1 & -4.5 & M1T57 & $\mathbf{2 4 - 2 8}$ & 1 & -6.5 & M1T74 & $\mathbf{3 0}$ & 1 & -8.6 \\
M2T40 & $\mathbf{8 - 1 2}$ & 2 & -3 & M2T57 & $\mathbf{2 4}$ & 2 & -4.5 & M2T74 & $\mathbf{3 6}$ & 2 & -6.0 \\
M4T40 & - & 4 & -1.9 & M4T57 & $\mathbf{2 2 - 2 8}$ & 4 & -3 & M4T74 & $\mathbf{3 2}$ & 4 & -4.1
\end{tabular}

to explain their observed vertical scale. In this section, the vertical mode selection in our idealized problem is discussed with the aid of very high resolution numerical simulations. Then, a detailed comparison between simulation and observation is presented to emphasize the MRG wave destabilization as a plausible formation mechanism for the observed EDJs.

\section{a. Vertical scale selection and intraseasonal frequency}

To adress the question of vertical scale selection, a second set of numerical simulations (Table 2) has been performed with the same equatorial basin configuration (rectangular $30^{\circ} \times$ $30^{\circ}$, flat bottom $h_{0}=5000 \mathrm{~m}$, constant stratification) but with different vertical modes and different periods of monochromatic forcing. In an attempt to be free of dissipative/diffusive effect, these simulations have been conducted with a very high spatial resolution $\left(1 / 11^{\circ}\right.$ and 25 or $12.5 \mathrm{~m}$ ) which require a vectorized and parallelized code, ROMS (Shchepetkin and McWilliams, 2005).

Simulation M2T40 is equivalent to the previous simulation SW, performed with SPEM. Instantaneous equatorial zonal velocity obtained in M2T40 (Fig. 8, left) matches SW (Fig. 2a) with vertical mode 8 visually dominant. The vertical mode spectrum of this zonal velocity field (Fig. 9, left) confirms that this circulation is dominated by modes 8 and 12, as with SW. Dynamics of the simulated jets appear similar in both models ${ }^{9}$. The vertical scale selection in SW is therefore independent of the large dissipation used in SPEM.

Figure 8's three panels allow one to visualize the effect of the forcing period on the vertical scale of the jets obtained in simulations with the same forcing vertical mode. From left to right, the three plotted simulations M2T40, M2T57 and M2T74 correspond to a forcing period of 40, 57 and 74 days respectively. Obviously, the longer the forcing period, the higher the vertical mode of the jets.

For simulations of nondimensional zonal wavenumbers $\hat{k}=-3,-4.5$ and -6 of excited MRG waves (Fig. 8), the vertical modes spectrum of the jets is dominated by the vertical modes 8-12, 24 and 36 respectively (solid line in Fig. 9). These values match closely the prediction of the analytical model of MRG wave destabilization (Hua et al., 2007), which are 12, 24 and 40 respectively. These simulations therefore confirm that in a forced case,

9. The temporal behavior of M2T40 jets has been verified to be the same in terms of equatorial basin modes, as in simulation SW. 

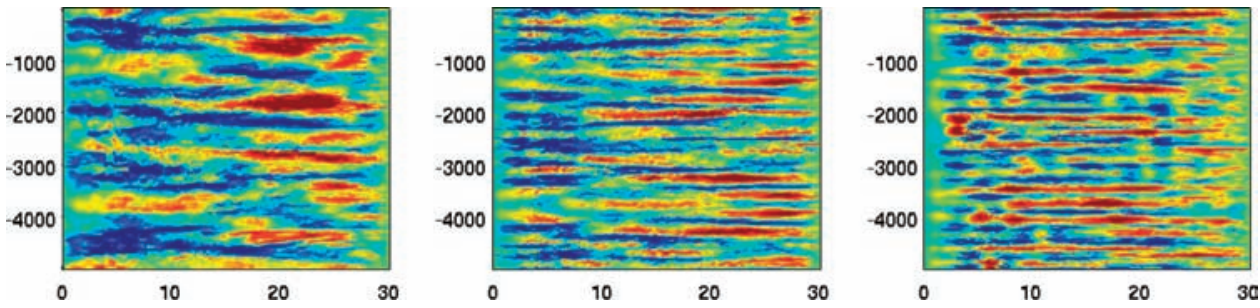

Figure 8. Equatorial zonal velocity after 10 years of simulations in M2T40, M2T57 and M2T74 (left to right), i.e. forcing with a vertical mode 2 and a period of 40, 57 and 74 days respectively. For each panel the colors range from $-U_{\max }$ (blue) to $U_{\max }$ (red), where $U_{\max }$ is the zonal velocity absolute value maximum.

the vertical mode which dominates the zonal circulation is still the most unstable baroclinic mode of the short MRG wave destabilization.

One of the most striking results of this second set of numerical simulations is that the dominant vertical scale of the equatorial zonal circulation seems to be independent of the forcing vertical mode (Fig. 10), but not of the forcing period (Fig. 8). Vertical mode spectrum (Fig. 9) reveals that simulations with a forcing period of 40 days have peaks around vertical modes 8 and 12; those with a forcing period of 57 days have vertical modes around 24; those with a forcing period of 74 days have vertical modes around 30 and 40 . The dimensional vertical scale selection depends only on the dimensional forcing period and not on the forcing vertical mode.

The analytical model of MRG wave destabilization in a channel predicts that a vertical scale of the zonal equatorial jets will be $p_{J}$-times smaller than that of the MRG wave with approximately $p_{J} \propto \hat{k}^{2}$. For short MRG waves $|\hat{k}|>>1$, their frequency is $\omega^{\star} \sim-\beta / k^{\star}$ (where $k^{\star}$ is their dimensional wavenumber). Therefore the dimensional vertical mode $p_{J}^{\star} \equiv p_{J} m^{\star}$ will be

$$
p_{J}^{\star} \propto T^{\star 2}
$$
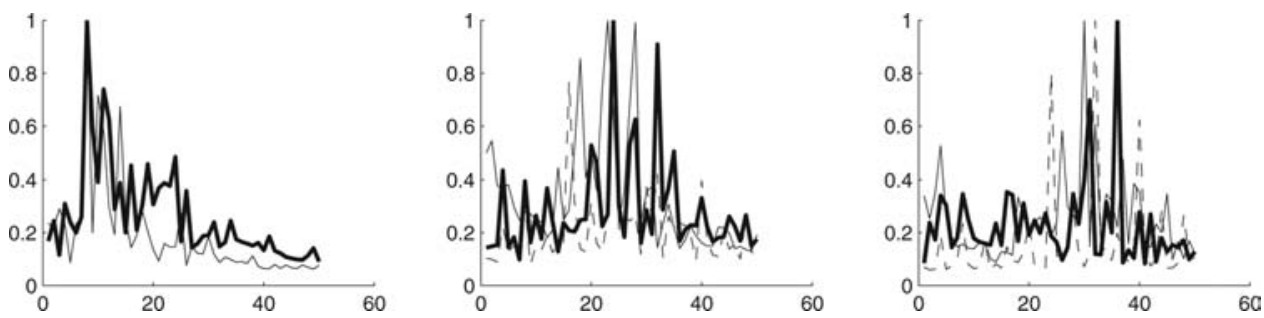

Figure 9. Zonally averaged vertical mode spectrum of Figure 8 simulations (thick solid line) as well as simulations with the same period of forcing but a vertical mode 1 (thin solid line) or 4 (dashed line). Each spectra amplitude is nondimensionalized by its maximum. M4T40 spectrum is not plotted, because no zonal jets are obtained (see text for details). 

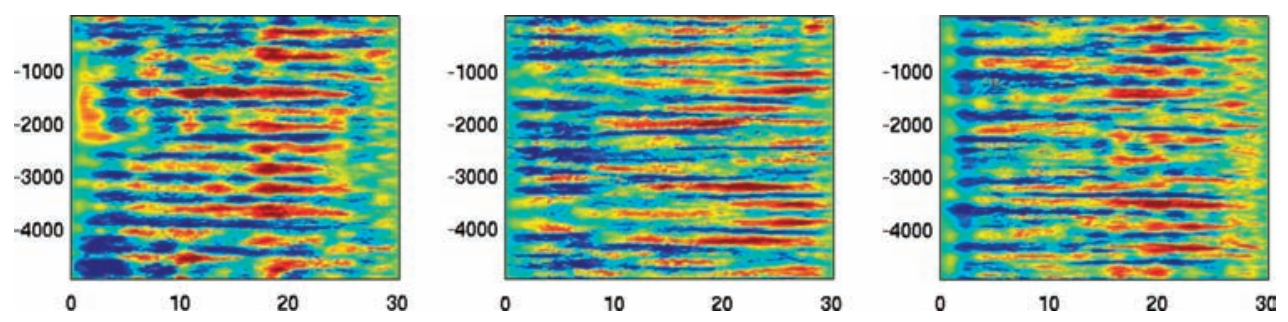

Figure 10. Equatorial zonal velocity after 10 years of simulations in M1T57, M2T57 and M4T57 (left to right), i.e. forcing with a period of 57 days and with a vertical mode 1, 2 and 4 respectively. For each panel the colors range from $-U_{\max }$ (blue) to $U_{\max }$ (red), where $U_{\max }$ is the zonal velocity absolute value maximum.

This analytic model predicts therefore that, in the short MRG wave limit, the vertical mode of the jets depends only on the period, and is independent of the vertical mode of the MRG wave. For the three different forcing periods used (Fig. 9), this approximated formula predicts a mode 12 for 40 days (left), a mode 24 for 57 days (middle) and a mode 40 for 74 days (right) - nearly what is obtained in the simulations.

However, this destabilization occurs only for short MRG waves. For instance, no jets were obtained in simulation M4T40 (this is why its vertical mode spectrum has not been plotted in the left panel of Fig. 9). This simulation corresponds to $\hat{k}=-1.9$ which is stable to shear instability. The case $\hat{k} \simeq-2$ is marginal between the shear instability and the triadic resonance (Section 2a). Therefore, such MRG waves could be destabilized by weakly nonlinear interactions due to a triadic resonance but only if the basin width is large enough (Section 2b). This simulation with no jets provides a reminder that in a short basin MRG waves can be responsible for high vertical mode baroclinic zonal jets only if they are in a sufficiently nonlinear regime, i.e. with a sufficiently short zonal scale. The previous statement can therefore be made slightly more accurate: vertical modes of the obtained zonal equatorial jets depend only on the forcing period and not on the vertical mode, provided that the excited MRG waves are sufficiently short (thus unstable).

Ultimately, one difference between channel and basin geometry is in the barotropic circulation. In both cases, an equatorially-trapped barotropic zonal circulation is created by MRG wave destabilization but, whereas it prevails in the entire domain for very short MRG wave in a channel, it stays confined near the western boundary in the forced basin simulation. Therefore, the direct nonlinear interactions which generate the barotropic circulation can also be significant in a basin, but only near the western boundary where the instability occurs because long zonal scale equatorially trapped barotropic signals propagate only westward ${ }^{10}$. The barotropic signal cannot propagate eastward into the interior basin, whereas the baroclinic jets do.

10. From the midlatitude dispersion relation, barotropic Rossby waves can only propagate westward if their meridional wavenumber is larger than their zonal wavenumber. 


\section{b. Comparison with observations}

From the analytical model and the simulations presented above, vertical scales of the EDJs observed in the Atlantic (around vertical mode 20) could be forced by a 60 days period. Therefore, this subsection presents a comparison of the jets obtained in simulation M2T57 with the observed EDJs.

The EDJs are zonal currents of alternate directions at the equator (Fig. 11). One of their characteristics is their strongly nonlinear dynamics. In order to compute their Froude number in the observations (Fig. 11), a vertical mode decomposition of the zonal velocity is used to separate EDJs from the low-mode component. The EDJs variance peak is near vertical mode 20 with a variance minimum at mode 10 . Consequently, vertical modes lower than 10 and the barotropic mode are substracted to the total field to obtain the relative velocity amplitude of EDJs (see Firing, 1987, for Pacific EDJs). The residual appears to have local values between 10 and $20 \mathrm{~cm} \cdot \mathrm{s}^{-1}$. For the long gravity wave speed of their dominant vertical mode $20\left(c_{20} \sim 20 \mathrm{~cm} . \mathrm{s}^{-1}\right)$, the computed Froude number exceeds 0.5 . A similar exercise for M2T57 gives a Froude number up to 0.5. It was demonstrated that the jets amplitude depends on the forcing amplitude (Section 4c, Fig. 6). Simulated jets can thus be as strongly nonlinear as observed EDJs.

The nonlinear character of the observed EDJs and of the simulated jets can also be inferred from their different eastward and westward currents meridional structure (Figs. 11 and 12). Eastward jets have a velocity maximum strongly localized in latitude near the equator (Kelvin-like meridional structure), whereas the westward jets are latitudinally wider and associated with two eastward extra-equatorial jets near 2N and 2S (Rossby-like). These distinct features have been previously noticed in observations (Gouriou et al., 1999) and correspond to a meridional homogeneity of the angular momentum (Hua et al., 1997; Bourles et al., 2003). Such homogenization of angular momentum leads to zero values of

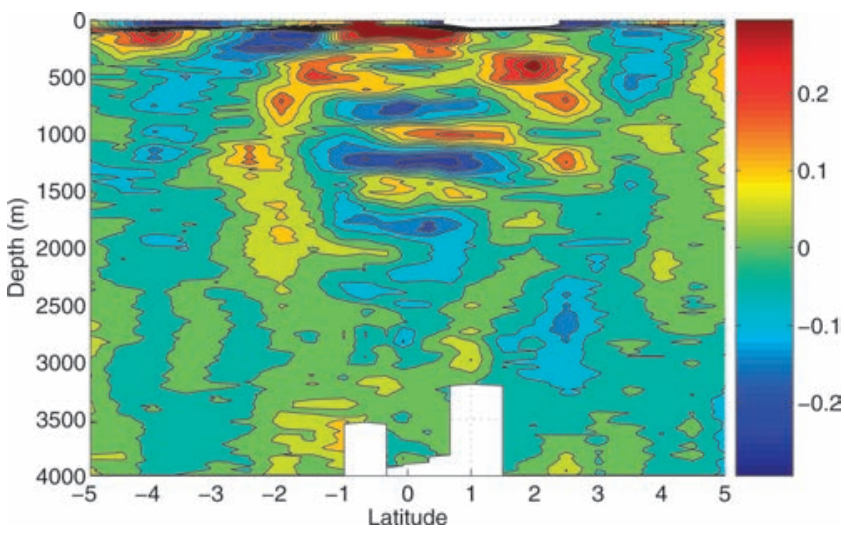

Figure 11. Meridional section of zonal velocity at 23W from the EQUALANT campaign (Gouriou et al., 2001). Solid contours every $5 \mathrm{~cm} \cdot \mathrm{s}^{-1}$. 


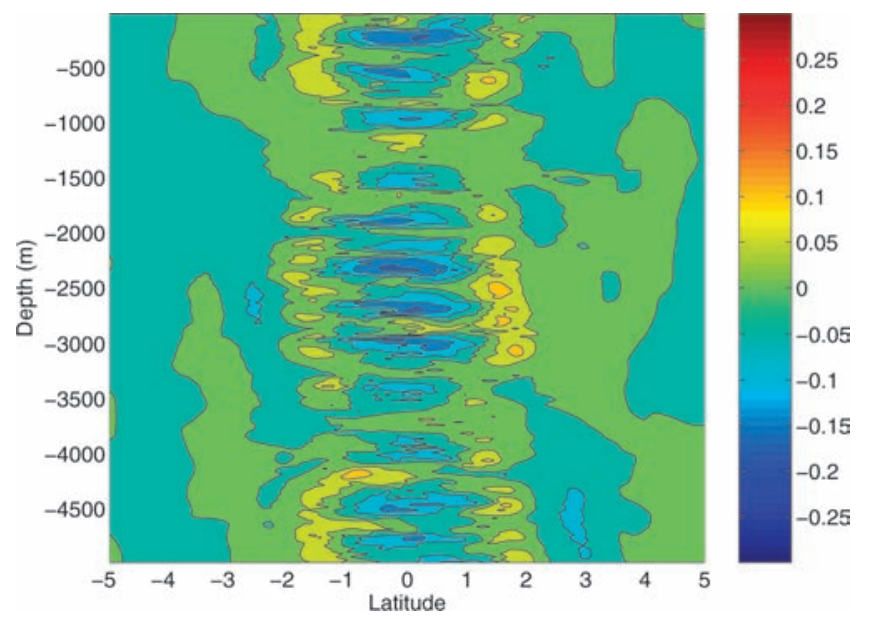

Figure 12. Meridional section of zonal velocity at $\mathrm{x}=6.6^{\circ}$ for simulation M2T57. Solid contours every $5 \mathrm{~cm} \cdot \mathrm{s}^{-1}$.

Potential Vorticity (PV) at the depths of westward jets, in simulation M2T57 and in the observations (Figs. 13 and 14). In the observations (Fig. 13), the vertical gradient of PV is due to the variable strength of stratification with depth, while the simulations have a constant stratification and therefore no large vertical scale PV gradient (Fig. 14). Even so, the meridional nonlinearities of simulated jets can be as strong as that of the observed EDJs.

Simulated jets zonal extent (see Fig. 2b) is also comparable with the observed one which can reach the scale of the basin (Schmid et al., 2003). Simulated jets time scales are set by their vertical modes, the basin width and the selected equatorial basin modes. In the

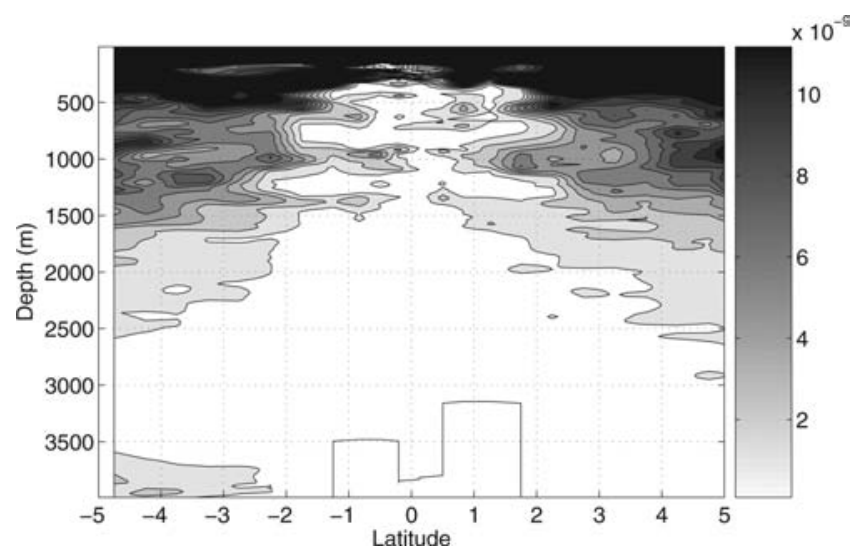

Figure 13. Meridional section of Potential Vorticity magnitude approximated as $\left|\left(f-U_{y}\right) \times g \rho_{z} / \rho_{0}\right|$ at $23 \mathrm{~W}$ from the EQUALANT campaign. 


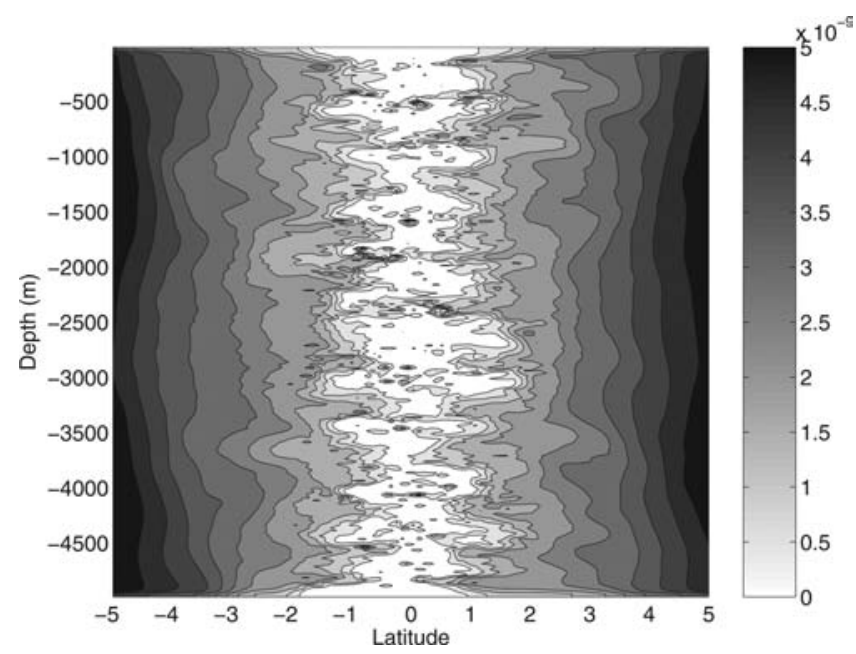

Figure 14. Meridional section of Ertel Potential Vorticity magnitude at $\mathrm{x}=9.8^{\circ}$ for simulation M2T57.

Atlantic, the first equatorial basin mode of EDJs vertical mode 20 is around five years. This low-frequency period is compatible with that derived from available in situ observations (Ponte and Luyten, 1989; Johnson and Zhang, 2003).

The last striking resemblance between simulated and observed zonal circulation is the belt of eastward-flowing current surrounding the jets. This belt is located around $+/-1.5^{\circ}$ with barotropic and low vertical mode structure (Figs. 11 and 12). In the simulations this eastward current belt arises directly from MRG wave destabilization, but as noted earlier, the barotropic part of this belt is trapped near the western boundary. Although this belt is also observed in the middle ot the Pacific (Firing, 1987), the simulations suggest that MRG wave destabilization can be part of its rationalization.

\section{Discussion}

Above, we have presented numerical results of basin-wide strongly baroclinic equatorial zonal jets with spatial and dynamical features comparable to Equatorial Deep Jets (EDJs) observed in the Atlantic Ocean. The jets arise from a time oscillating, large vertical scale, western boundary current. This inter-hemispheric oscillations excite mixed Rossby-gravity (MRG) wave trains propagating along the equator with the same vertical mode. Their subsequent destabilization leads to the higher vertical mode equatorial jets.

MRG wave destabilization has been extensively studied with initial value problems in a channel geometry and found to arise from shear instability (Hua et al., 2007). In the permanently forced basin simulations presented here, this instability is zonally localized near the western boundary, but produces zonal jets in the whole equatorial basin. The spacial and temporal variability of these jets corresponds to low-frequency equatorial basin modes 
(Cane and Moore, 1981; Yang and Liu, 2003). Basin modes with more complex vertical and zonal propagation and boundary reflection are found when the forcing amplitude is increased. Basin equatorial modes together with vertical propagation have also been seen in recent numerical simulations of an idealized equatorial Atlantic Ocean (Ascani et al., 2006).

As predicted by Hua et al. (2007)'s idealized analytic model, it was found that the simulated vertical scale is mainly a function of the forcing frequency and is independent of the forcing vertical mode (as long as the excited MRG waves are in a sufficiently nonlinear regime $)^{11}$. To reproduce the Atlantic EDJs vertical scale with this mechanism, a forcing period of approximately 60 days is required. Because this period is typical of the deep western boundary current in the equatorial Atlantic Ocean (Johns et al., 1993; Fischer and Schott, 1997), the formation mechanism of baroclinic jets presented here appears to be a likely explanation of the observed Atlantic EDJs. To reproduce Indian or Pacific EDJs vertical scale, forcing periods around 60 and 100 days respectively would be required, and are found in the deep western boundary current variability poleward of the equator (Warren et al., 2002; Kawabe et al., 2005).

This EDJs formation mechanism provides a rationale for the observed vertical scale and a known energy source to explain highly nonlinear dynamics in the deep equatorial ocean. This mechanism remains compatible with previous equatorial wave theories (Eriksen, 1981; Ponte and Luyten, 1989) and does not exclude a possible role of inertial instability in angular momentum homogenization of westward jets (Hua et al., 1997). Inertial instability at smaller vertical scales than the EDJs can actually take place in the westward jets, enhancing the angular momentum homogenization without selecting the vertical scale of the jets (d'Orgeville et al., 2004).

The observed meridional structure of the zonal currents in the equatorial Atlantic includes a vertically continuous, extra-equatorial belt of eastward currents centered about $2 \mathrm{~N}$ and $2 \mathrm{~S}$. Simulations reported here reproduce this pattern near the western boundary. MRG waves destabilization can therefore be part of the explanation but other mechanisms may also come into play. For instance, the observed depth-variable stratification background is expected to enhance low vertical mode zonal circulation and to favor the observed confinement of the jets above $2500 \mathrm{~m}$ depth. Simulations with less idealized configurations (variable Brunt-Vaisala frequency, non monochromatic oscillatory forcing, existence of the Equatorial Under Current, a mean western boundary current, etc.) should allow to more realistically reproduce the deep equatorial zonal circulation.

The case of a mean interhemispheric western boundary current has been previously investigated, using also extra-equatorial forcing (Kawase et al., 1992). The obtained equatorial circulation has the same vertical scale as the forcing and is a transient spin-up adjustment

11. Note that the vertical scale selection depends only on the destabilization process and is therefore also independent of the size of the basin (as seen in simulations with different basin length, which will be reported elsewhere). 
as predicted by linear theory (Kawase, 1987). In contrast, the case of a purely oscillating western boundary current reported here leads to a permanent bifurcation at the equator with smaller vertical scales. The interhemispheric character of this oscillation is fundamental in order to excite MRG waves with their subsequent destabilization into high vertical mode zonal jets. In the simulations, a purely antisymmetric forcing in a symmetric geometry was designed to excite only MRG waves. Such waves emerge easily at the equator with a time oscillating source as soon as the symmetry is broken, for instance by a slanted western boundary (Moore and McCreary, 1990).

In comparison to Kawase et al. (1992)'s results, a time-oscillating western boundary current appears to be essential for a permanent bifurcation along the equator. Oscillating forcings have already been explored to attempt to explain the equatorial bifurcation revealed by tongues of tracer extrema (e.g. Weiss et al., 1985). In a shallow water equatorial basin model, the nonlinear response to a Rossby wave which is forced at the eastern boundary is confined to the western boundary (Thompson and Kawase, 1993) and has, by construction, the same vertical scale as that of the forcing. Li et al. (1996) have examined the case of an oscillatory forcing which is asymmetric about the equator in a shallow water equatorial basin model: they propose that the chaotic mixing due to the combination of intraseasonal MRG waves and an annual Rossby wave can explain the equatorial bifurcation of the $\mathrm{CFC} /$ salinity maximum tongue. Again, the shallow water model setup implies that the equatorial response has the same vertical scale as that of the forcing. Here we emphasize that MRG wave destabilization, and thus a high vertical mode bifurcation as observed, requires vertical modes interaction. Moreover, transport due to nonlinear baroclinic zonal jets is more effective than chaotic mixing in explaining tracer tongues along the equator.

Acknowledgments. This work has benefited from grant 51777 from IDRIS. Access to the Earth Simulator is through IFREMER-CNRS-ES MOU. We would like to thank S. Le Gentil for her development in ROMS and F. Pinsard for her help in completing the simulations on the ES. We would like to acknowledge very helpful conversations with P. Klein and R. Schopp. BLH acknowledges support from the French ANR (Agence Nationale pour la Recherche), contract no. ANR 05 CIGC 010.

\section{REFERENCES}

Andrié, C., J. F. Ternon, M. J. Messias, L. Memery, B. Bourlès, Y. Gouriou, and C. Oudot. 1998. Chlorofluoromethane distributions in the deep equatorial Atlantic during January-March 1993. Deep-Sea Res. I, 45, 903-930.

Ascani, F., D. Wang and E. Firing. 2006. Equatorial deep jets in a simple ocean generation circulation model. Eos Trans. AGU, 87(36), Ocean Sci. Meet. Suppl., Abstract OS33C-05.

Bourlès, B., C. Andrié, Y. Gouriou, G. Eldin, Y. du Penhoat, S. Freudenthal, B. Dewitte, F. Gallois, R. Chuchla, F. Baurand, A. Aman and G. Kouadio. 2003. The deep currents in the eastern equatorial Atlantic Ocean. Geophys. Res. Lett., 30, doi:10.1029/2002GL015095.

Bunge, L., C. Provost, J. M. Lilly, M. d'Orgeville, A. Kartavtseff and J.-L. Melice. 2006. Variability of the horizontal velocity structure in the upper $1600 \mathrm{~m}$ of the water column on the equator at $10^{\circ} \mathrm{W}$. J. Phys. Oceanogr., 36, 1287-1304.

Cane, M. A. and D. W. Moore. 1981. A note on low-frequency equatorial basin mode. J. Phys. Oceanogr., $11,1578-1584$. 
Dengler, M. and D. Quadfasel. 2002. Equatorial deep jets and abyssal mixing in the Indian Ocean. J. Phys. Oceanogr., 32, 1165-1180.

d'Orgeville, M., B. L. Hua, R. Schopp and L. Bunge. 2004. Extended deep equatorial layering as an imprint of inertial instability. Geoph. Res. Lett., 31, L22303, doi: 10.1029/2004GL020845.

Eriksen, C. C. 1981. Deep currents and their interpretation as equatorial waves in the western Pacific Ocean. J. Phys. Oceanogr., 11, 48-70.

1982. Geostrophic equatorial deep jets. J. Mar. Res., 40, 143-157.

Firing, E. 1987. Deep zonal currents in the central equatorial Pacific. J. Mar. Res., 45, 791-812.

Fischer, J. and F. A. Schott. 1997. Seasonal transport variability of the deep western boundary current in the equatorial Atlantic. J. Geophys. Res., 102, 27751-27769.

Furue, R. 2001. An equatorial diffusive boundary layer and the equatorial stacked jets in thermally driven OGCMs. J. Phys. Oceanogr., 31, 2737-2748.

Gouriou, Y., C. Andrié, B. Bourlès, S. Freudenthal, S. Arnault, A. Aman, G. Eldin, Y. du Penhoat, F. Baurand, F. Gallois and R. Chuchla. 2001. Deep circulation in the equatorial Atlantic Ocean. Geophys. Res. Lett., 28, 819-822.

Gouriou, Y., B. Bourlès, H. Mercier and R. Chuchla. 1999. Deep jets in the equatorial Atlantic Ocean. J. Geophys. Res., 104, 21217-21226.

Haidvogel, D. B., J. L. Wilkins and R. E. Young. 1991. A semi-spectral primitive equation ocean circulation model using vertical and orthogonal curvilinear horizontal coordinates. J. Comput. Phys., 94, 151-185.

Hua, B. L., M. d'Orgeville, C. Menesguen and H. Sasaki. 2007. Destabilization of mixed Rossbygravity waves and equatorial zonal jets formation. J. Fluid Mech. (submitted).

Hua, B. L., D. W. Moore and S. Le Gentil. 1997. Inertial nonlinear equilibration of equatorial flows. J. Fluid Mech., 331, 345-371.

Johns, W. E., D. M. Fratantoni and R. J. Zantopp. 1993. Deep western boundary current variability off northeastern Brazil. Deep-Sea Res. I, 40, 293-310.

Johns, W. E., T. E. Lee, F. A. Schott, R. J. Z. Kess and R. H. Evans. 1990. The North Brazil current retroflection: seasonal structure and eddy variability. J. Geophys. Res., 95, 22103-22120.

Johnson, G. C., E. Kunze, K. E. M. Taggart and D. W. Moore. 2002. Temporal and spatial structure of the equatorial deep jets in the Pacific Ocean. J. Phys. Oceanogr., 32, 3396-3407.

Johnson, G. C. and D. Zhang. 2003. Structure of the Atlantic Ocean equatorial deep jets. J. Phys. Oceanogr., 33, 600-609.

Kawabe, M., D. Yanagimoto, S. Kitagawa and Y. Kuroda. 2005. Variations of the deep western boundary current in Wake island passage. Deep-Sea Res. I, 52, 1121-1137.

Kawase, M. 1987. Establishment of deep ocean circulation driven by deep-water production. J. Phys. Oceanogr., 17, 2294-2317.

Kawase, M., L. M. Rothstein and S. R. Springer. 1992. Encounter of a deep western boundary current with the equator: a numerical spin-up experiment. J. Geophys. Res., 97, 5447-5463.

LaCasce, J. H. and J. Pedlosky. 2004. The instability of Rossby basin modes and the oceanic eddy field. J. Phys. Oceanogr., 34, 2027-2041.

Leetma, A. and P. F. Spain. 1981. Results from a velocity transect along the equator from $125^{\circ} \mathrm{W}$ to $159^{\circ}$ W. J. Phys. Oceanogr., 11, 1030-1033.

$\mathrm{Li}, \mathrm{X}$. and P. Chang. 1999. Mixing induced by the Atlantic equatorial wave activity in an eddy-resolving OGCM. J. Geophys. Res., 104, 13303-13315.

Li, X., P. Chang and R. C. Pacanonowski. 1996. A wave-induced stirring mechanism in the mid-depth equatorial ocean. J. Mar. Res., 54, 487-520.

Luyten, J. R. and J. C. Swallow. 1976. Equatorial undercurrents. Deep-Sea Res., 23, 999-1001. 
Moore, D. W. and J. P. McCreary. 1990. Excitation of intermediate-frequency equatorial waves at a western ocean boundary: With application to observations from the Indian Ocean. J. Geophys. Res., 95, 5219-5231.

Ponte, R. M. 1989. A simple model for deep equatorial zonal currents forced at lateral boundaries. J. Phys. Oceanogr., 19, 1881-1891.

Ponte, R. M. and J. R. Luyten. 1989. Analysis and interpretation of deep equatorial currents in the central Pacific. J. Phys. Oceanogr., 19, 1025-1038.

Richardson, P. L. and D. M. Fratantoni. 1999. Float trajectories in the deep western boundary current and deep equatorial jets of the tropical Atlantic. Deep-Sea Res II, 46, 305-333.

Ripa, P. 1981. On the theory of nonlinear wave-wave interactions among geophysical waves. J. Fluid Mech., 103, 87-115.

Schmid, C., B. Bourlès and Y. Gouriou. 2005. Impact of the deep equatorial jets on the zonal transport in the Atlantic. Deep-Sea Res. II, 52, 409-428.

Schmid, C., Z. D. Garaffo, E. Johns and S. L. Garzoli. 2003. Pathways and variability at intermediate depths in the tropical Atlantic, in Interhemispheric Water Exchange in the Atlantic Ocean, G.J. Goni and P. Malanotte. Rizzoli, eds., Elsevier, 503 pp.

Send, U., C. Eden and F. Schott. 2002. Atlantic equtorial deep jets: space-time structure and crossequatorial fluxes. J. Phys. Oceanogr., 32, 891-902.

Shchepetkin, A. and J. McWilliams. 2005. The regional oceanic modeling system: a split-explicit, free-surface, topography-following-coordinate ocean model. Ocean Model., 9, 891-902.

Suga, T. and L. D. Talley. 1995. Antarctic Intermediate Water circulation in the tropical and subtropical south Atlantic. J. Geophys. Res., 100, 13441-13453.

Thompson, L. and M. Kawase. 1993. The nonlinear reponse of an equatorial ocean to oscillatory forcing. J. Mar. Res., 51, 467-496.

Wang, D. 1995. On thermally forced deep equatorial circulation in a GCM. J. Phys. Oceanogr., 25, $2155-2165$.

Warren, B. A., T. Whitworth and J. H. LaCasce. 2002. Forced resonant undulation in the deep Mascarene basin. Deep Sea Res. II, 49, 1513-1526.

Weisberg, R. H. and A. M. Horigan. 1981. Low-frequency variability in the equatorial Atlantic. J. Phys. Oceanogr., 11, 913-920.

Weiss, R. F., J. L. Bullister, R. H. Gammon and M. J. Warner. 1985. Atmospheric chlorofluoromethanes in the deep equatorial Atlantic. Nature, 314, 608-610.

Wüst, G. 1935. Schichtung Zirkulation des Atlantischen Ozeans: die Stratosphäre. In Wissenschftliche Ergebnisse der Deutschen Atlantischen Expedition auf dem Forschungs- und Vermessungsschift "Meteor" 1925-1927, 6, $180 \mathrm{pp}$.

Yang, H. and Z. Liu. 2003. Basin modes in a tropical-extratropical basin. J. Phys. Oceanogr., 33, 2751-2763.

Received: 23 March, 2005; revised: 26 October, 2006. 